\title{
B-vitamin deficiency in patients treated with antiepileptic drugs.
}

\author{
Scott Mintzer \\ Thomas Jefferson University \\ Christopher T Skidmore \\ Thomas Jefferson University \\ Michael R Sperling \\ Thomas Jefferson University
}

\section{Follow this and additional works at: https://jdc.jefferson.edu/neurologyfp \\ Part of the Neurology Commons \\ Let us know how access to this document benefits you}

\section{Recommended Citation}

Mintzer, Scott; Skidmore, Christopher T; and Sperling, Michael R, "B-vitamin deficiency in patients treated with antiepileptic drugs." (2012). Department of Neurology Faculty Papers.

Paper 48.

https://jdc.jefferson.edu/neurologyfp/48

This Article is brought to you for free and open access by the Jefferson Digital Commons. The Jefferson Digital Commons is a service of Thomas Jefferson University's Center for Teaching and Learning (CTL). The Commons is a showcase for Jefferson books and journals, peer-reviewed scholarly publications, unique historical collections from the University archives, and teaching tools. The Jefferson Digital Commons allows researchers and interested readers anywhere in the world to learn about and keep up to date with Jefferson scholarship. This article has been accepted for inclusion in Department of Neurology Faculty Papers by an authorized administrator of the Jefferson Digital Commons. For more information, please contact: JeffersonDigitalCommons@jefferson.edu. 


\author{
As submitted to: \\ Epilepsy and Behavior \\ And later published as: \\ B-Vitamin Deficiency in Patients Treated with Antiepileptic \\ Drugs \\ Volume 24, Issue 3, July 2012, Pages 341-344 \\ DOI: 10.1016/j.yebeh.2012.04.132
}

Scott Mintzer, MD, Christopher T. Skidmore, MD, Michael R. Sperling, MD

Jefferson Comprehensive Epilepsy Center, Department of Neurology, Thomas Jefferson University, Philadelphia, PA

Corresponding author: Dr. Mintzer, 900 Walnut Street, Suite 200, Philadephia PA 19107; e-mail scott.mintzer@jefferson.edu; phone 215-955-1222; fax 215-955-0606 


\section{ABSTRACT}

Enzyme-inducing antiepileptic drugs (AEDs) produce many alterations in metabolism, including vitamin levels. Whether they produce clinically-relevant deficiency of B vitamins has rarely been assessed. We obtained B vitamin levels in patients who were being converted from an inducing AED (phenytoin or carbamazepine) to a non-inducing AED (levetiracetam, lamotrigine, or topiramate), with measurements both before and $\geq 6$ weeks after the switch. A group of normal subjects underwent the same studies. Neither folate nor B12 deficiency was seen in any patient. Vitamin B6 deficiency was found in 16/33 patients (48\%) taking inducers, compared to $1 / 11$ controls ( $9 \%$; $\mathrm{p}=0.031)$. After switch to non-inducers, only 7 patients ( $21 \%)$ were B6 deficient $(\mathrm{p}=0.027)$. The incidence of deficiency was similar regardless of which inducing or non-inducing AED was being taken. Our findings demonstrate that treatment with inducing AEDs commonly causes pyridoxine deficiency, often severe. This could conceivably contribute to the polyneuropathy sometimes attributed to older AEDs, as well as other chronic heath difficulties. 


\section{INTRODUCTION}

Enzyme-inducing antiepileptic drugs (AEDs) produce a considerable number of metabolic alterations, including changes in serum lipids, hormones, bone turnover, and various vitamin levels [1]. Some of these parameters, such as cholesterol, are appropriately examined in a continuous fashion, as their contribution to disease is graded. In contrast, other measures are best examined in a dichotomous fashion, as their contribution to clinical disease is generally felt to depend upon whether a patient's level declines below a certain critical threshold.

The B vitamins folate, cyanocobalamin (B12), and pyridoxine (B6) belong to the latter category. A number of studies have examined the effects of enzyme-inducing and other AEDs on these vitamins, but most have looked at them as continuous variables [2-4]. This approach is less than ideal for determining clinical relevance in some respects, as a patient whose vitamin level declines a bit due to AED therapy will suffer no clinical consequences as long as that level remains in the range that will appropriately support normal function. Whether AEDs produce frank deficiency of vitamins has been little-studied. B6 deficiency in epilepsy patients has been the subject of only a single uncontrolled study[5]. One large study did examine folate and B12 levels in a dichotomous fashion, finding that deficiency of one or both these vitamins was more common in patients taking a number of AEDs, including many inducers (phenytoin, carbamazepine, primidone), some newer drugs with limited enzyme-inducing capacity (oxcarbazepine, topiramate) and several non-inducers (gabapentin, pregabalin, valproate) [6]. This study was limited by its cross-sectional design, reducing its ability to attribute these deficiencies to the effects of drugs. These authors did not examine B6 levels.

The present study assessed B vitamin deficiency in a group of patients transitioning from an older, enzyme-inducing AED to a newer generation AED. The crossover design, together with a normal control group, permits attribution of drug effects. Our goal was to determine whether inducing AEDs produce clinically relevant deficiency of B vitamin levels. 


\section{METHODS}

We studied patients with epilepsy on CBZ or PHT monotherapy whose treatment regimen was being changed to monotherapy with one of the newer agents LEV, LTG, or TPM. Patients were participating in one of two studies designed to examine the effects of AED conversion on lipids and other vascular risk factors [7, 8]. Any patient undergoing such a drug switch was asked to participate. Patients had been taking the former drugs for at least one month, and the decision to alter therapy was made by the patient's treating physician for clinical reasons (e.g., side effects, inadequate seizure control, or concerns about chronic AED use). These patients were compared to the same group of normal subjects utilized in our previous investigation[7]. The drug-treated patients provided a fasting blood sample during therapy with the initial drug, and a second sample following AED conversion, at least 6 weeks after the inducing agent had been discontinued. Target doses of AEDs were generally low: $500 \mathrm{mg}$ BID of LEV, 100 - $200 \mathrm{mg}$ BID of LTG, and 50-75 mg BID of TPM (except for a single patient taking TPM at $150 \mathrm{mg}$ BID). In the normal subjects, two fasting blood samples were taken 10 weeks apart (comparable to the the anticipated inter-sample time in the drug-treated patients, whose inducer would be gradually tapered over several weeks).

Blood samples were analyzed for folate, pyridoxal-5' -phosphate (B6), and B12 levels in serum, as well as the level of the AED the patient was taking at the time. We excluded from the present analyses any patients with conditions which might be expected to have a major impact upon nutrient absorption, such as pernicious anemia, celiac disease, active inflammatory bowel disease, or prior bariatric surgery. In addition, we excluded patients who had a major change in health state during the study period (e.g. pregnancy) which might be expected to alter vitamin levels over the course of the study. Subjects taking high-dose B vitamin supplements were also excluded. Those taking multivitamins were found to have B6 levels which were markedly higher than all other subjects at baseline, and as a consequence were excluded from B6 analyses. Folate and B12 levels in these latter patients at baseline did not differ from those of the other subjects. Some individual measurements were not carried out in certain samples due to laboratory or handling errors. The total number of patients was $n=28$ for folate, $n=29$ for $B 12$, and $n=33$ for B6; for normal subjects $n=14$ for folate and B12 and $n=11$ for B6. Vitamin levels were 
performed by Quest Diagnostics (San Juan Capistrano, CA). Folate and B12 were measured using chemiluminescent immunoassay with a total allowable error of $30 \%$ and coefficients of variation of $6.0-8.3 \%$ and $3.3-9.0 \%$ respectively. B6 was measured in normal subjects and in the majority of the patients using a radioenzyme assay method with a coefficient of variation of 13.3-14.4\% and lower limit of quantitation of $2.5 \mathrm{ng} / \mathrm{mL}$. The laboratory subsequently updated its technique for this measure, such that in the remainder of the patients B6 was measured using liquid chromatography and tandem mass spectometry with a lower quantitation limit of 2.0 $\mathrm{ng} / \mathrm{mL}$ and a total allowable error of $25 \%$. The correlation between the two B6 detection methods is $>97 \%$. Comparison of B vitamin deficiency in drug-treated and normal subjects was performed using the Fisher's Exact or Chi-squared tests. The incidence of B6 deficiency in drugtreated patients before and after drug switch was analyzed using the McNemar test. Analyses were performed using InStat 3.0 (GraphPad, San Diego, CA).

\section{RESULTS}

The epilepsy patients averaged 44 years of age (range 18-88) and were $57 \%$ female. With regard to ethnicity, the group was approximately $77 \%$ Caucasian, $13 \%$ of African descent, $7 \%$ Latino, and 3\% from other ethnic groups. They had been treated with their initial medication for anywhere between 30 days and 35 years. Those taking CBZ had serum levels ranging from 1.9$16.6 \mu \mathrm{g} / \mathrm{mL}$ (mean: 8.7), with all but one patient having a level > 4.0. Among those taking PHT, levels ranged from 3.3 - $28.9 \mu \mathrm{g} / \mathrm{mL}$ (mean: 15.1); two patients had levels of 3.3 and 5.1, with all the rest having levels $\geq 8.9$. The total time between the two blood draws ranged from 42 to 308 days (mean: 123). The normal subjects not taking AEDs who served as comparators were described in a previous publication[7]. This group was similar in age, gender, and race to the patient cohort.

Using a threshold value of $<3 \mathrm{ng} / \mathrm{ml}$, folate deficiency was not seen in any of the patients during treatment with the older AEDs, nor when they were switched to the newer AEDs. A 
single PHT-treated patient had a folate level right at the cutoff $(3 \mathrm{ng} / \mathrm{ml})$, and this increased to $3.5 \mathrm{ng} / \mathrm{ml}$ after switch to a newer AED. Thus, while folate levels were lower during inducer treatment (data not shown), this did not produce threshold deficiency. None of the normal subjects were folate deficient at either blood draw.

With regard to B12 deficiency, using the standard cutoff value of $<200 \mathrm{pg} / \mathrm{ml}$, there was no B12 deficiency seen among any of the epilepsy patients, either under treatment with an inducing AED or under treatment with a non-inducing drug. In fact, there was more B12 deficiency among the normal subjects: none were deficient at the first draw, though several were close to the cutoff, and 3 actually declined into the deficient range at the time of the second draw. Thus, we found no evidence of clinically-relevant B12 deficiency among AED-treated epilepsy patients.

Results with respect to B6 deficiency are shown in Figure 1. Using the standard cutoff of $5 \mathrm{ng} / \mathrm{ml}, 16$ of 33 epilepsy patients (48\%) were B6 deficient, as compared to 1/11 normal subjects (9\%). B6 deficiency was significantly more common at baseline in the inducer-treated patients than in the normal subjects (Fisher's Exact, $\mathrm{p}=0.031$ ). The degree of B6 deficiency was not trivial, with 10 of these 16 patients, or $30 \%$ of the entire cohort, having levels at or below the limit of detection of the predominant assay $(\leq 2.5 \mathrm{ng} / \mathrm{ml})$. In fact, using that number as a stringent cutoff, B6 deficiency remained more common in patients than in controls (Fisher's Exact, p= 0.046). Using the more lenient threshold of $<7.5 \mathrm{ng} / \mathrm{ml}$, the high prevalence of deficiency remained a trend (Fisher's Exact, $\mathrm{p}=0.096$ ). Following switch to a non-inducing AED, B6 deficiency was seen in 7 of 33 epilepsy patients (21\%), a significant decline from the $48 \%$ incidence in those same patients during inducing-drug treatment (McNemar, p=0.027) (Fig.1).

The findings do not appear meaningfully related to the specific assay used, as B6 deficiency was seen in $12 / 27$ patients (44\%) using the radioenzyme assay and in $4 / 6$ patients $(66 \%)$ using the newer mass spectrometry assay. The findings also do not appear related to the specific inducing or non-inducing AED used, as B6 deficiency was seen in 7 of 12 patients while taking PHT (58\%) and in 9 of 21 patients taking CBZ (43\%). When PHT- and CBZ-treated patients were considered as separate groups and compared to normal subjects, the incidence of B6 deficiency remained significant (Chi-squared, $\mathrm{p}=0.046$ ). Once crossed-over to a non-inducing 
AED, B6 deficiency appeared in nearly-identical proportions regardless of whether the patient was switched to LEV, LTG, or TPM (Figure 2). 


\section{DISCUSSION}

A number of studies have examined B vitamin levels in AED-treated epilepsy patients, generally in the context of their potential contribution to homocysteine levels. As a consequence, the majority of these studies have been concerned with the magnitude and statistical significance of any serum level changes, since changes throughout the range of values may impact upon homocysteine levels. Yet for most other clinical concerns, changes in vitamin levels within the normal range are unlikely to be clinically relevant. For this reason, we undertook to specifically examine the issue of whether treatment with enzyme-inducing AEDs contributes to threshold deficiency of B vitamins.

The major result of the present investigation was the finding that B6 deficiency is very common in the inducer-treated population, and in most cases was improved by switch to a noninducer. Several studies have found reduced B6 levels in CBZ-treated patients, and in two of the studies this could clearly be attributed to CBZ [2, 4, 9]. However, none of these studies reported the incidence of B6 deficiency, so that the clinical relevance of these changes, other than their potential contribution to homocysteine deficiency, remained uncertain. Only one previous study examined B6 deficiency in a categorical fashion, finding as we did that the majority of PHT- and CBZ- treated patients were B6 deficient; however, this study did not include a control group[5]. Thus, to our knowledge, ours is the first study to demonstrate that frank B6 deficiency is more common in CBZ- and PHT-treated patients with epilepsy than in control subjects, and that this deficiency is very likely caused or exacerbated by the drugs themselves.

Furthermore, the degree of B6 deficiency in these patients was remarkable, with $30 \%$ of the entire inducer-treated cohort having B6 levels that were undetectable or nearly so. Relatively little is known about the consequences of B6 deficiency, as it is distinctly uncommon in adults. Among the most well-established clinical consequences of B6 deficiency is peripheral neuropathy, generally encountered in the context of treatment with the antitubercular drug isoniazid, which forms a complex with B6 and thus reduces its availability to tissues[10]. It is standard clinical practice to supplement isoniazid-treated patients with B6 to avoid the depletion 
that can otherwise occur via this mechanism. This neuropathic action of isoniazid, and the present findings, are of interest in light of the evidence that PHT may be neuropathic[11, 12] . There is also evidence that other inducing AEDs may contribute to the development of peripheral neuropathy $[13,14]$. Further clinical investigation is warranted to assess the possibility that this could be due to B6 deficiency. Deficiency of B6 may also contribute to anemia and may increase the risk of a number of cancers[15]. In addition, there is the well-documented genetic condition of B6-dependent epilepsy in infants[16]. It is not clear that B6 deficiency contributes to seizures in adults or older children lacking the relevant mutation, though a recent case series suggested B6 deficiency as a cause of new-onset refractory seizures in several critically ill patients[17]. Specific study would be required to determine whether the kind of extreme B6 deficiency seen here might contribute to chronic AED-resistant epilepsy.

The mechanism for B6 deficiency caused by inducing AEDs remains unclear. B6 is oxidized in the liver prior to urinary excretion[15], and such oxidizing enzymes are frequent targets of enzyme inducers, so that it is likely that increased activity of the oxidizing enzyme in the presence of PHT or CBZ results in increased catabolism of B6. Another alternative is that, like isoniazid, these drugs form a complex with B6 and result in increased urinary excretion. We are not aware of any pharmacologic investigation of these hypotheses.

The noteworthy negative findings in our study involved the absence of folate or B12 deficiency in the AED-treated epilepsy patients. The findings with regard to B12 are in good agreement with those of Linnebank et al [6], who found that B12 deficiency was uncommon in inducer-treated patients (or in patients taking any AED), and no more common than in healthy controls. Thus, while both those authors and our group [7] found that inducers appear to lower B12 levels, it is unlikely that this effect is of clinical significance. With regard to folate, those authors found that about a quarter of CBZ- or PHT-treated patients were folate deficient, a finding which contrasts starkly with our data. Both studies used the same cutoff for folate deficiency, and only one of our patients in whom folate was measured was taking a multivitamin supplement. It is possible that differences in diet could explain the folate discrepancy, as folate supplementation of foodstuffs is common in the United States, but not in Germany. This points up a limitation of our study, and indeed of virtually all studies in this area: absence of knowledge 
regarding subjects' nutritional intake. Dietary assessments are, in general, difficult and of dubious accuracy due to poor subject recall, inability to corroborate food intake, and other factors. This makes the use of our repeated measures design particularly valuable, as it is unlikely that subjects would have experienced major changes in diet that would have meaningfully affected vitamin levels during the period between the two blood draws; furthermore, any tendency to alter diet would have been reflected equally in both patient and control groups. Thus, even with this limitation, our study points to the effects of inducing AEDs as being causative of B6 deficiency. It is also worth noting the ability of folate supplementation to mask functional B12 deficiency, a phenomenon which has been specifically reported in the epilepsy population[18]. While this could conceivably have been a factor in our cohort, the results with respect to B12 were similar to those seen in the study done in Germany, where there is little folate supplementation of food. Thus, we feel this is unlikely to have been a meaningful contributor to our negative findings with respect to B12.

In summary, our data reveal a high prevalence and degree of B6 deficiency in patients under treatment with inducing AEDs. While we cannot exclude that some of this might relate to the underlying epilepsy, the design of our study makes it clear that PHT and CBZ are responsible for the lion's share of this finding. Further work is also needed to ascertain the potential clinical impact of B6 deficiency in this population with regard to peripheral neuropathy, homocysteinemia, seizures, or other metabolic consequences. 


\section{FIGURE LEGENDS}

\section{Figure 1: Vitamin B6 deficiency during treatment with various AEDs}

Bars represent the fraction of patients deficient during the period of treatment with a given inducing AED (shown in green) or non-inducing AED (in blue). Patient numbers were $\mathrm{n}=12$ for phenytoin (PHT), n=21 for carbamazepine (CBZ), n=9 for levetiracetam (LEV), $n=13$ for lamotrigine (LTG), and $\mathrm{n}=11$ for topiramate (TPM).

Figure 2: Vitamin B6 deficiency in epilepsy patients and in normal subjects

Green bars represent the fraction of patients deficient at baseline, while blue bars represent the fraction of patients deficient at the second draw. ${ }^{*} \mathrm{p}<0.05$ (Fisher's Exact) for comparison of epilepsy patients vs. normal controls at baseline (draw 1). $\dagger \mathrm{p}<0.05$ (McNemar) for comparison of epilepsy patients at baseline (while taking an inducing drug) and at draw 2 (after switch to newer-generation drug). 


\section{REFERENCES}

1. Mintzer S. Metabolic consequences of antiepileptic drugs. Curr Opin Neurol. 2010

2. Schwaninger M, Ringleb P, Winter R et al. Elevated plasma concentrations of homocysteine in antiepileptic drug treatment. Epilepsia. 1999;40 (3):345-350.

3. Sener U, Zorlu Y, Karaguzel O, Ozdamar O, Coker I, Topbas M. Effects of common antiepileptic drug monotherapy on serum levels of homocysteine, vitamin B12, folic acid and vitamin B6. Seizure. 2006;15 (2):79-85.

4. Verrotti A, Pascarella R, Trotta D, Giuva T, Morgese G, Chiarelli F. Hyperhomocysteinemia in children treated with sodium valproate and carbamazepine. Epilepsy Research. 2000;41 (3):253-257.

5. Tamura T, Aiso K, Johnston KE, Black L, Faught E. Homocysteine, folate, vitamin B-12 and vitamin B-6 in patients receiving antiepileptic drug monotherapy. Epilepsy Res. 2000;40 (1):7-15.

6. Linnebank M, Moskau S, Semmler A et al. Antiepileptic drugs interact with folate and vitamin B12 serum levels. Ann Neurol. 2011;69 (2):352-359.

7. Mintzer S, Skidmore CT, Abidin CJ et al. Effects of antiepileptic drugs on lipids, homocysteine, and C-reactive protein. Ann Neurol. 2009;65 (4):448-456.

8. Mintzer S, Skidmore CT, Rankin SJ et al. Conversion from enzyme-inducing antiepileptic drugs to topiramate: Effects on lipids and c-reactive protein. Epilepsy Res. 2012;98 (1):8893.

9. Attilakos A, Papakonstantinou E, Schulpis K et al. Early effect of sodium valproate and carbamazepine monotherapy on homocysteine metabolism in children with epilepsy. Epilepsy Res. 2006;71 (2-3):229-232.

10. Zhang Y. Tuberculosis. In: Waldman SA, Terzic A, eds. Pharmacology and therapeutics: principles to practice. Philadelphia: Saunders; 2009:1089-1108.

11. Lovelace RE, Horwitz SJ. Peripheral neuropathy in long-term diphenylhydantoin therapy. Archives of neurology. 1968;18 (1):69.

12. Ramirez JA, Mendell JR, Warmolts JR, Griggs RC. Phenytoin neuropathy: structural changes in the sural nerve. Ann Neurol. 1986;19 (2):162-167.

13. Baldini S, Carenini L, Leone M, D’Alessandro G, Bottacchi E. Peripheral neuropathy caused by antiepileptic drugs. Neurophysiological study of the $\mathrm{A} \delta$ and $\mathrm{C}$ fibers. The Italian Journal of Neurological Sciences. 1992;13 (3):233-238.

14. Shorvon SD, Reynolds EH. Anticonvulsant peripheral neuropathy: a clinical and electrophysiological study of patients on single drug treatment with phenytoin, carbamazepine or barbiturates. Journal of Neurology, Neurosurgery \& Psychiatry. 1982;45 (7):620-626.

15. Spinneker A, Sola R, Lemmen V, Castillo MJ, Pietrzik K, Gonzalez-Gross M. Vitamin B6 status, deficiency and its consequences--an overview. Nutr Hosp. 2007;22 (1):7-24.

16. Mikati MA, Trevathan E, Krishnamoorthy KS, Lombroso CT. Pyridoxine-dependent epilepsy: EEG investigations and long-term follow-up. Electroencephalogr Clin Neurophysiol. 1991;78 (3):215-221.

17. Gerlach AT, Thomas S, Stawicki SP, Whitmill ML, Steinberg SM, Cook CH. Vitamin B6 
deficiency: a potential cause of refractory seizures in adults. JPEN J Parenter Enteral Nutr. 2011;35 (2):272-275.

18. Drazkowski J, Sirven J, Blum D. Symptoms of B12 deficiency can occur in women of child bearing age supplemented with folate. Neurology. 2002;58 (10):1572-1573. 